\title{
Microscopic Mechanism of Water Diffusion in Glucose Glasses
}

\author{
Valeria Molinero and William A. Goddard III \\ Materials and Process Simulation Center, Division of Chemistry and Chemical Engineering, \\ California Institute of Technology (MC 139-74), Pasadena, California 91125, USA
}

(Received 26 October 2004; published 21 July 2005)

\begin{abstract}
The preservation of biomaterials depends critically on the mobility of water in the glassy state, manifested as a secondary $\beta$ relaxation and diffusion. We use coarse grain simulations to elucidate the molecular mechanism underlying the relaxations for water-glucose glass, finding two pathways for water diffusion: (i) water jumps into neighbor water positions (linking to water structure), and (ii) water jumps into glucose positions (coupling to glucose rotation). This work suggests strategies for enhancing preservation by stiffening the segmental motions of the carbohydrates.
\end{abstract}

DOI: 10.1103/PhysRevLett.95.045701

The formation of carbohydrate glasses is sought for the preservation of foodstuff, pharmaceuticals, and cryopreserved proteins and cells, as a means to achieve textural and chemical stability. However, recent studies of carbohydrate solutions have shown that vitrification is not sufficient to arrest water mobility: water can still diffuse in the glassy carbohydrate matrices [1-5], decreasing the chemical stability of the material [6].

Dielectric relaxation studies of glucose and its water mixtures also show the existence of mobility below the glass transition, manifested as a strong secondary $\beta$ relaxation $[7,8]$. This secondary relaxation in the glass is another evidence of the existence of relaxation modes decoupled from the viscosity, even in the absence of water. The microscopic origin of secondary relaxations in glasses is still disputed $[9,10]$. There is no consensus on whether its origin is a local restricted reorientational process that involves all molecules [10-12] or a spatially heterogeneous process that encompasses big rotational motions of molecules located in mobile regions [9].

The diffusion of small molecules or ions in glasses is not unique to carbohydrate mixtures but also seen in glassy ionic conductors [13], metallic glasses [14], and polymers [15]. In all these cases, the translational mobility of the small molecule or ion decouples from that of the glassy matrix. Whether the goal is to increase the decoupling (as in ionic conductors) or to decrease it (as in matrices used for preservation, like carbohydrates), there is a need to understand the microscopic mechanism that allows such decoupling in the glass. This Letter addresses the question of what mechanisms of relaxation operate in water-glucose glasses to account for the $\beta$ relaxation and the decoupling of water translation from the sugar matrix.

Molecular dynamics (MD) simulations provide the level of detail required to elucidate these microscopic mechanism. Nevertheless, the study of glassy molecular mixtures by MD has been hampered by the computational cost of atomistic simulations. We study here the relaxation of a $12.2 \%$ water-glucose (WG) glass using our recently developed M3B coarse grain model [16] for carbohydrate-water mixtures. M3B represents the water molecule by one bead
PACS numbers: 64.70.Pf, 61.20.Ja, 61.20.Lc, 61.25.Em

or pseudoatom and glucose by three beads as shown in Fig. 1 and is $\sim 7500$ faster than atomistic MD, extending the simulation time to the order of a microsecond. Despite its simplicity, M3B predicts water distributions in glucose in excellent agreement with full atomistic simulations [17], glass transition temperatures in accordance with experiment [16], and a jump-diffusion mechanism and Arrhenius temperature dependence for water diffusion in supercooled glucose mixtures that agrees with all experimental results known for water in concentrated carbohydrates mixtures [17].

In this Letter we determine the mechanism of diffusion of water in glucose, the canonical carbohydrate, and show that the diffusion of water in the glass is related to the distribution of water in the mixture through the existence of facilitated position exchanges between neighbor water molecules, and by the rotation of the glucose molecule, that also persists below the glass transition.

We studied the diffusion in glucose glass at $T / T_{g}=$ 0.92. For this mixture, $T_{g}^{\mathrm{M} 3 \mathrm{~B}}=239 \mathrm{~K}[16]\left(T_{g}^{\mathrm{exp}}=\right.$ $240 \mathrm{~K}$ [18]). The simulation methods and preparation of the $12.2 \mathrm{wt} \%$ WG system are described in Ref. [17]. The glass was prepared from the $12.2 \mathrm{wt} \% \mathrm{WG}$ supercooled mixture at $250 \mathrm{~K}$ of Ref. [17], cooling it to $220 \mathrm{~K}$, and then running isothermal isobaric MD for $0.85 \mu \mathrm{s}$ at $220 \mathrm{~K}$. The results presented here correspond to the last $750 \mathrm{~ns}$ of that trajectory.



FIG. 1. Mapping of coarse grain beads (balls) on the atomistic model of glucose molecule (cylinders). Water is represented by a single bead. See Ref. [16] for interaction parameters. 
The existence of diffusion in the glass was investigated through the calculation of the van Hove self-correlation function $G_{s}(r, t)$ for the translation of water and glucose centers of mass, $4 \pi r^{2} G_{s}^{a}(r, t)=\frac{1}{N_{a}}\left\langle\sum_{i=1}^{N_{a}} \delta\left(r-\mid \vec{r}_{i}(t)-\right.\right.$ $\left.\left.\vec{r}_{i}(0) \mid\right)\right\rangle$. This function indicates the probability for each component ( $\mathrm{W}$ or $\mathrm{G}$ ) to travel a distance $r$ in a time interval $t$. The inset of Fig. 2(a) shows the distribution of distances traveled by water and glucose at $T / T_{g}=0.92$ after $t=$ $0.5 \mu \mathrm{s}$. The two components display strikingly different behavior: glucose shows a single peak in the distribution, that does not evolve noticeably with time, indicating that - as expected - there is no translational motion of the sugar in the glass. Water, on the contrary, remains mobile and presents multiple peaks in $4 \pi r^{2} G_{s}(r, t)$. The characteristic time for water to diffuse to a first neighbor
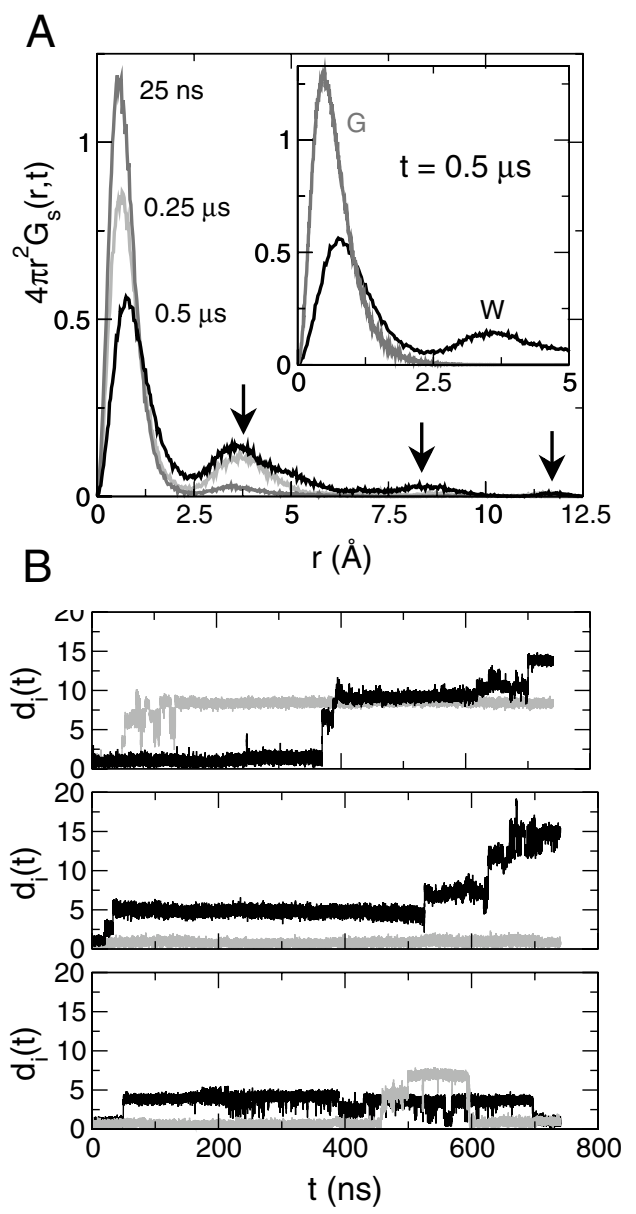

FIG. 2. (a) Water is mobile in the glucose glass, and diffuse through jumps. The characteristic time for water diffusion to a first neighbor position was estimated to be $\sim 1.8 \mu$ s from the SE fit of the decay up to $0.5 \mu \mathrm{s}$ of the intermediate scattering function $F s\left(k=1.875 \AA^{-1}, t\right)$ obtained by Fourier transforming $G s(r, t)$. Inset: Decoupling of water $(\mathrm{W})$ and glucose $(\mathrm{G})$ translation in the glass: $\mathrm{G}$ center of mass does not translate, while $\mathrm{W}$ is still mobile. (b) Individual water displacements $d_{i}(t)=$ $\left|\boldsymbol{r}_{i}(t)-\boldsymbol{r}_{i}(0)\right|$ in the glass consist of well-defined jumps. Water has a heterogeneous dynamics. About $1 / 4$ of the molecules jump, stay $\sim 1 \mathrm{~ns}$, and then return. position was estimated to be $1.8 \mu \mathrm{s}$, and its diffusion coefficient to be $D=l^{2} / 6 \tau=1.0 \times 10^{-14} \mathrm{~m}^{2} / \mathrm{s}$, which agrees with $D \sim 10^{-14} \mathrm{~m}^{2} / \mathrm{s}$ measured for water in $10 \mathrm{wt} \%$ and $6 \mathrm{wt} \%$ water-maltose mixtures at their respective glass transition temperatures [1,2]. This diffusivity is 9 orders of magnitude higher than the Stokes-Einstein prediction at $T_{g}, D \sim 10^{-23} \mathrm{~m}^{2} / \mathrm{s}$.

The existence of multiple peaks and their nonevolving positions [Fig. 2(a)] suggest that water diffuses by displacements of $\sim 3.5 \AA$ (size of water) in the translationally frozen carbohydrate matrix. This is indeed seen in the displacement of individual water molecules, $d_{i}(t)=$ $\left|r_{i}(t)-r_{i}(0)\right|$ [Fig. 2(b)]: the water molecules rattle around the same position for a long - and widely variable - time, and then jump $\sim 3.5 \AA$ away.

Water relaxation shows a stretched exponential (SE) decay with $\beta=0.59$, for $k=2 \pi / 3.35 \AA$. This $\beta$ corresponds to a half width of $\sim 2$ decades in the frequency domain [19], as observed for the electrical relaxation of ionic conducting glasses [13]. This result stresses the commonality between relaxations of small diffusive particles-water, ions, or atoms - in glasses.

Having established that the center of mass of glucose does not translate in the glass and that water displaces through jumps, we analyze the factors that allow the decoupling of water diffusion from carbohydrate translation. It has been suggested that water may diffuse through free volume (FV) channels formed by packing the sugars [2,5]. To examine this hypothesis, we computed the FV available to spherical probes with radius ranging from $R_{p}=0$ to $1.5 \AA$ using a grid based method [20]. We find that the FV network in $12.2 \% \mathrm{WG}$ percolates for a probe radius of $R_{p \text { perc }} \sim 0.5 \AA$, much smaller than the size of water. The same $R_{p \text { perc }}$ was found for water-sucrose mixtures with low water content (10-33 wt \%), using atomistic simulations [20]. Contrary to observations for glassy polymers [21], there are no free volume channels available to facilitate water diffusion in the glass. Hence, the observed water motion implies concerted displacements with either water molecules or glucose beads.

Water diffusion in the glass proceeds through jumping into the position of neighbor water and glucose beads. For every jump made by a water molecule, we identified which particle occupied the new water position before the jump. We found that most (68\%) of the jumps involve water replacing a neighbor water molecule, with the other important contribution (22\%) from water jumping into glucose smallest bead, B6 (see Table I). Moreover, the small

TABLE I. Percentage of positions to which water jumps in glucose glass, and Morse radius parameters of beads $(R)$.

\begin{tabular}{lcccc}
\hline \hline & $\mathrm{W}$ & $\mathrm{B} 6$ & $\mathrm{~B} 4$ & $\mathrm{~B} 1$ \\
\hline \% W jumps & 68 & 22 & 4 & 6 \\
$\mathrm{R}(\AA)$ & 3.77 & 4.63 & 6.11 & 5.13 \\
\hline \hline
\end{tabular}


beads jump more frequently than large ones: water makes a jump every $39 \mathrm{~ns}$, glucose's B6 every $50 \mathrm{~ns}$, while the bigger B 1 and B4 average only 2.1 and 0.1 jumps in $750 \mathrm{~ns}$, respectively. Although we identified jumps in the motion of the individual glucose beads, we find no sign of jumps or diffusion for the center of mass of the sugar molecule [Fig. 2(a)].

Water in the $12.2 \%$ WG mixture has a locally heterogeneous structure that we characterized by their number of first neighbor waters (distance $<4 \AA$ ) [17]. This neighbor distribution for the glassy mixture is shown in Fig. 3(a). Table I shows that most of the water molecules jump into a water neighbor position. We define [17] the water facilitation [Fig. 3(b)] as the ratio between the fraction of total jumps for a specific water coordination number and the fraction of waters having this coordination number [see Fig. 3(a)]. The water facilitation in the glass shows that the presence of water neighbors significantly increases the probability of water jumps. We find the same trend (but less dramatic) for water facilitation for the same system above the glass transition [17]. This increased importance of water structure on water dynamics below $T_{g}$ is due to decreased contribution from the other water relaxation channel involving the motion of glucose beads.

The existence of jumps for waters that lack water neighbors is consistent with the results of Table I, showing 32\% of jumps of water into glucose bead positions. The absence of translation of the carbohydrate indicates that there must be a local motion of the sugar contributing to the dynamics of water. In the M3B model, these modes correspond to glucose rotation.

To obtain additional mechanistic detail, we computed the autocorrelation of glucose reorientations [using angles defined by two unit vectors: the "backbone vector" $\boldsymbol{r}_{B}$ in the direction that connects the beads $\mathrm{B} 1$ and $\mathrm{B} 4$, and the "side vector" $\boldsymbol{r}_{S}$ that connects the middle of $\boldsymbol{r}_{B}$ to the position of B6, that represents the side group of atomistic glucose (see Fig. 1)]. The autocorrelation of the corresponding angles $\theta^{m}(t)=\boldsymbol{r}_{m}(t) \cdot \boldsymbol{r}_{m}(0)$ is defined by $4 \pi \sin (\theta) P^{m}(\theta, t)=\frac{1}{N_{G}}\left\langle\sum_{i=1}^{N_{G}} \delta\left(\theta-\left[\theta_{i}^{m}(t)-\theta_{i}^{m}(0)\right]\right)\right\rangle$.


FIG. 3. (a) Heterogeneity of the local structure of water, characterized through the distribution of the number of closest water neighbors (squares). Circles show distribution of neighbors for water just before they jump. (b) Facilitation is defined as the ratio between these two probabilities. The presence of water neighbors enhances the jump probability.
Figure 4 shows that the backbone and side rotations both proceed through big jumps that appear as secondary peaks in the autocorrelation function. The probability of backbone jumps is lower than for side jumps, in agreement with Table I. The rotational characteristic times from the partial decay of the first order rotational autocorrelation function up to $0.5 \mu \mathrm{s}$ leads to values of roughly $\tau_{S} \sim 3 \mu \mathrm{s}$ and $\tau_{B} \sim 15 \mu \mathrm{s}$. Thus glucose rotational times are somewhat longer than for water translation, $\tau_{W} \sim 1.8 \mu \mathrm{s}$.

The persistence of glucose rotation in the glass and the existence of rotational jumps agree with 2D NMR experiments on $15 \mathrm{wt} \% \mathrm{WG}$ just above $T_{g}$ [22]. The contribution of continuous diffusion (small jumps) to the rotational relaxation is insignificant, as can be seen in the invariant position of the first peak of $P(\theta, t)$ in Fig. 4. Thus, we find that the most relevant mechanism for the rotation of glucose in the glass is through big jumps. We expect the magnitude of the angle jumps obtained with the coarse grain model to be larger than for the fully atomistic system, reflecting the lack of "sticky points" present atomistically (e.g., hydrogen bonds) but not in the M3B model. The M3B model probably has a smoother energy landscape than the atomistic model.

Our results indicate the translation of water and the rotation of the sugar both contribute to the relaxation in the glass, and thus to the observed $\beta$ relaxation. In the glass both water translation and glucose rotation occur through big jumps with comparable characteristic times: $\tau_{W} \sim$ $1.8 \mu \mathrm{s}, \tau_{S} \sim 3 \mu \mathrm{s}$, and $\tau_{B} \sim 15 \mu \mathrm{s}$, which explains the observation of a single broad $\beta$ peak in the dielectric relaxation of water-glucose mixtures [7]. We expect that water rotation - meaningless in our coarse grain modelwould also contribute to the relaxation. Our results indicate that the broadening of the $\beta$ relaxation of glucose by the addition of $10 \%$ water [7] is due to the contribution of water translation occurring with comparable time scales to glucose rotation. This also explains the observed increased in amplitude of the $\beta$ peak with the addition of water to glucose [7].

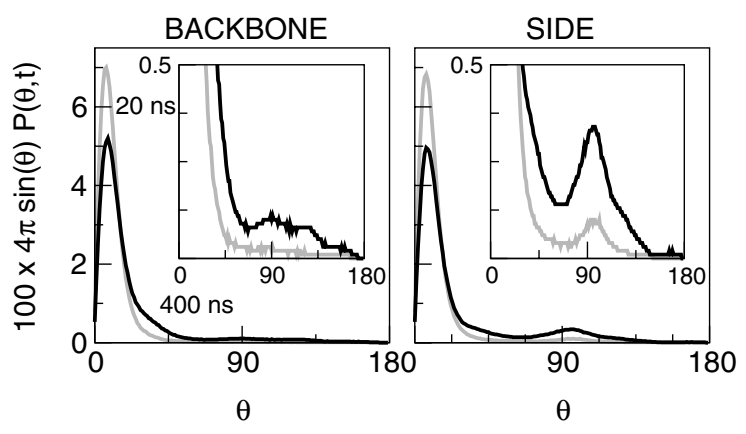

FIG. 4. Reorientation of backbone and side vectors of glucose molecules (see text) show that glucose rotation in the glass is nondiffusive and occurs through big jumps. Insets zoom on the corresponding figures. The black and gray curves correspond to $t=400 \mathrm{~ns}$ and $20 \mathrm{~ns}$, respectively. 
We find that the water and glucose molecules that jump are clustered. However, our simulation times $(\sim 1 \mu \mathrm{s})$ are too short to support a spatially heterogeneous scenario in the glass. (For $T / T_{g}=1.05$ we find that all water and glucose molecules contribute to the relaxation in a $t=$ $0.65 \mu$ s simulation.) Note that, in the glass, the mobile glucose molecules are mobile only with respect to their rotation: Not even the ones that display high rotational mobility show any sign of translational diffusion.

With respect to the microscopic mechanism of water diffusion in the glass, we conclude that water mobility decouples from the translation of the sugar, though it is still facilitated by the local dynamics of the carbohydrate, also persistent below $T_{g}$. There are no free volume channels that facilitate water motion in this glass, and water diffusion occurs through two relaxation pathways: (i) water jumping into a neighbor water position, and (ii) water jumping into a neighbor glucose bead position. The former couples the water dynamics to its local structure, and the latter couples to the rotational dynamics of the carbohydrate, in particular, to the rotation that involves the exchange of water with the small "side group" B6 of glucose. The facilitation of water dynamics by water neighbors reflects the difference in time scales of the two processes leading to water diffusion: increased water neighbors favors the first, more probable (see Table I), pathway. The lower probability of the second pathway reflects the hindrance to the rotation of a more complex topology formed by bigger particles in a dense mixture (see above the lower jump probability of the glucose beads). Based on this mechanism for water diffusion, we expect that water mobility in carbohydrate solutions will be more sensitive to the glass transition for lower water content mixtures, where the water facilitation mechanism is less relevant and water mobility relies on its coupling to the dynamics of the sugar matrix. An NMR study of water mobility in maltose for water contents in the range 520 wt \% confirms this prediction [4]. On the other hand, the existence of facilitated diffusion of water due to glucose rotation explains why water diffusion is observedalbeit very slow - in carbohydrate glasses even at very low water contents [2], below the percolation threshold for water in these systems [17]. An urgent need in biopreservation is to develop improved formulations that would decrease water mobility in the glass. This work indicates that local mobility of the carbohydrate is a key to control water diffusivity in low water content mixtures. This mechanism can be generalized to glasses of oligosaccharides, were the segmental motion of the monosaccharide units in the chain would provide the local modes that couple to water diffusion. In this respect, we find that the rigidity of the internal modes of the host molecule tunes the degree of water mobility: we observe a 13 -fold enhancement of water diffusion in supercooled $12 \%$ Wdodecamaltooligomer at $475 \mathrm{~K}$ when the torsional potentials that restrict the segmental dynamics of the polymer in
M3B are turned off [23]. In an analogous way, the lower diffusivity of water in trehalose than in sucrose [24] may be related to the higher conformational rigidity of the former disaccharide. The results presented here indicate that preservation mixtures with minimum water mobility should be designed by decreasing the water content and carbohydrate segmental mobility.

At the coarse grain level description of this work, we expect the results to be relevant to understand the mechanism of mobility of small species in other translationally frozen matrices, such as metallic [14] and ionic conductors glasses [13]. On the other hand, the success of M3B to describe the essential features and time scales of molecular transport in water-glucose solutions emphasizes that the explicit inclusion of hydrogen bonds, charges, or even atomic detail, is not essential to model these fully hydroxylated systems.

The facilities of the Materials and Process Simulation Center are supported by ONR-DURIP, ARO-DURIP. In addition the MSC is supported by MURI-ARO, MURIONR, DOE (ASC, FETL), NSF (CHE), NIH, Aventis Pharma, ChevronTexaco, Nissan Corp., Berlix Biopharma, and Beckman Institute.

[1] R. Parker and S.G. Ring, Carbohydr. Res. 273, 147 (1995).

[2] R. H. Tromp et al., Carbohydr. Res. 303, 199 (1997).

[3] B. P. Hills et al., Mol. Phys. 99, 1679 (2001).

[4] I. J. van den Dries et al., J. Phys. Chem. B 102, 10483 (1998).

[5] B. P. Hills et al., J. Mol. Liq. 63, 229 (1995).

[6] S. M. Lievonen et al., J. Food Sci. 67, 2100 (2002).

[7] R. K. Chan et al., J. Phys. Chem. 90, 6358 (1986).

[8] T. R. Noel et al., Carbohydr. Res. 282, 193 (1996).

[9] G. P. Johari, J. Non-Cryst. Solids 307, 317 (2002).

[10] T. Blochowicz and E. A. Rössler, Phys. Rev. Lett. 92, 225701 (2004).

[11] M. Vogel et al., J. Phys. Chem. B 104, 4285 (2000).

[12] H. Wagner et al., J. Phys. Chem. B 103, 4071 (1999).

[13] C. A. Angell, Chem. Rev. 90, 523 (1990); C. A. Angell et al., J. Appl. Phys. 88, 3113 (2000).

[14] X.P. Tang et al., Nature (London) 402, 160 (1999); F. Faupel et al., Rev. Mod. Phys. 75, 237 (2003); U. Geyer et al., Phys. Rev. Lett. 75, 2364 (1995).

[15] P. Medick et al., J. Magn. Reson. 159, 126 (2002).

[16] V. Molinero et al., J. Phys. Chem. B 108, 1414 (2004).

[17] V. Molinero et al., J. Phys. Chem. A 108, 3699 (2004).

[18] Y. Roos, Carbohydr. Res. 238, 39 (1993).

[19] R. Hilfer, Phys. Rev. E 65, 061510 (2002).

[20] The FV was averaged over 14 reconstructed [16] atomistic configurations, following the procedure of V. Molinero et al., Chem. Phys. Lett. 377, 469 (2003).

[21] M. L. Greenfield et al., Macromolecules 26, 5461 (1993).

[22] A. M. Wachner et al., J. Chem. Phys. 111, 10611 (1999).

[23] V. Molinero and W. A. Goddard III, "Decoupling of Water Mobility in Concentrated Carbohydrates" (to be published).

[24] N. Ekdawi-Sever et al., J. Phys. Chem. A 107, 936 (2003). 